\title{
THE BORDERING ALGORITHM AND PATH FOLLOWING NEAR SINGULAR POINTS OF HIGHER NULLITY*
}

\author{
HERBERT B. KELLER $\dagger$
}

\begin{abstract}
We study the behavior of the bordering algorithm (a form of block elimination) for solving nonsingular linear systems with coefficient matrices in the partitioned form $\left(\begin{array}{cc}C_{C}^{*} & B \\ D\end{array}\right)$ when $\operatorname{dim} \mathcal{N}(\boldsymbol{A}) \geqq 1$. Systems with this structure naturally occur in path following procedures. We show that under appropriate assumptions, the algorithm, which is based on solving systems with coefficient matrix $A$, works as $A$ varies along a path and goes through singular points. The required assumptions are justified for a large class of problems coming from discretizations of boundary value problems for differential equations.
\end{abstract}

Key words. path following, bordering algorithm, block elimination, singular systems

1. Introduction. We study here some specific procedures for solving linear systems of the special form

$$
\mathscr{A}\left(\begin{array}{l}
\mathbf{x} \\
\boldsymbol{\xi}
\end{array}\right) \equiv\left(\begin{array}{cc}
A & B \\
C^{T} & D
\end{array}\right)\left(\begin{array}{l}
\mathbf{x} \\
\boldsymbol{\xi}
\end{array}\right)=\left(\begin{array}{l}
\mathbf{g} \\
\boldsymbol{\gamma}
\end{array}\right) .
$$

The matrix $\mathscr{A}$ is of order $N+\nu$ with the indicated submatrices: $A$ of order $N, D$ of order $\nu, B$ is $N \times \nu$ and $C$ is $\nu \times N$. The vectors $\mathbf{x}, \mathbf{g} \in \mathbb{R}^{N}$ and $\xi, \gamma \in \mathbb{R}^{\nu}$. Such linear systems arise when using Newton's method in some specific path following algorithms to be discussed later. Indeed it is families of systems of the form (1.1) that we solve in applications and invariably $N \gg \nu$. In fact a major part of our current interest stems from the fact that in the course of this process the matrix $A$ becomes singular or near singular, while $\mathscr{A}$ remains nonsingular. The so-called bordering algorithm that we use to solve (1.1) when both $A$ and $\mathscr{A}$ are nonsingular, which is based on solving systems of the form

$$
A \mathbf{v}=\mathbf{b}, \quad \mathbf{v}, \mathbf{b} \in \mathbb{R}^{N},
$$

can be shown to be valid for our applications when $A$ is singular. (The bordering algorithm is but a special case of block Gaussian elimination.) Our analysis has been presented in [8] for the case $\nu=1$. However this is not generally available and it is somewhat surprising that the case $\nu \geqq 1$, treated here, can be done so simply. Thus the present study includes the results of [8] but is independent of that reference.

In $\S 2$ we formulate the bordering algorithm for solving (1.1) when $A$ and $\mathscr{A}$ are nonsingular. We also show how the Woodbury formula [4] can be used in this case but under more restrictive conditions.

In $\S 3$ we examine the solution of (1.1) when $A$ has nullity $\nu$ and $\mathscr{A}$ is nonsingular. We use here bases for the right and left null spaces of $A$. In $\S 4$ we show how these bases are obtained from an exact $L U$-decomposition (with pivoting) of $A$. Then in $\S 5$ we show how the bordering algorithm can be applicable to this singular case when a finite precision factorization is employed.

The $L U$-factorization of singular matrices must, in general employ full pivoting. However, for a large class of "banded" linear systems arising from discretizations of boundary value problems for differential equations, we show that partial pivoting can

* Received by the editors March 15, 1982, and in revised form July 26, 1982. This work was supported by the U.S. Department of Energy under contract EY-76-S-03-0767, Project agreement \#12, and the U.S. Army Research Office under contract DAAG 29-78-C-0011.

$\dagger$ Applied Mathematics, California Institute of Technology 217-50, Pasadena, California 91125. 
be used until half a bandwidth from the end; only then is full pivoting required. This is done in $\S 6$ where we also briefly describe the path following applications.

2. Bordering algorithms: nonsingular $\boldsymbol{A}$ and $\mathscr{A}$. Suppose $A$ and $\mathscr{A}$ are nonsingular. Then we can solve $(1.1)$ as follows. Determine the $N \times \nu$ matrix $V$ and the vector $\mathbf{w} \in \mathbb{R}^{N}$ from

$$
A V=B, \quad A \mathbf{w}=\mathbf{g}
$$

Find $\boldsymbol{\xi} \in \mathbb{R}^{\nu}$ by solving

$$
\left(D-C^{T} V\right) \xi=\gamma-C^{T} \mathbf{w}
$$

Then evaluate $\mathbf{x} \in \mathbb{R}^{N}$ as

$$
\mathbf{x}=\mathbf{w}-V \boldsymbol{\xi}
$$

This procedure is simply a form of block Gaussian elimination that results from the factorization

$$
\left(\begin{array}{cc}
A & B \\
C^{T} & D
\end{array}\right)=\left(\begin{array}{cc}
A & 0 \\
C^{T} & I
\end{array}\right)\left(\begin{array}{cc}
I & V \\
0 & D-C^{T} V
\end{array}\right)
$$

Since $A$ is nonsingular $V$ and $\mathbf{w}$ are uniquely defined by $(2.1 \mathrm{a}, \mathrm{b})$. Since in addition $\mathscr{A}$ is nonsingular the Schür complement of $A$ in $\mathscr{A}$ must be nonsingular and this is just

$$
D-C^{T} A^{-1} B=D-C^{T} V .
$$

Thus $\boldsymbol{\xi}$ is uniquely defined by (2.2). Using these results in (1.1) we see that the solution is obtained.

After an $L U$-factorization of $A$ we need only $\nu+1$ backsolves to obtain $V$ and w. Then we must solve the $\nu$ th order system (2.2). The inhomogeneous term in this system and the formation of $\mathbf{x}$ in (2.3) requires the equivalent of $2 \nu$ inner products of $N$-vectors. Thus for $N \gg \nu$, our main case of interest, the bulk of the work is in the factorization of $A$.

Another interesting procedure for solving (1.1) is to use the last $\nu$ equations to eliminate $\boldsymbol{\xi}$ from the first $N$ equations. This can be done if $D$ is nonsingular-a requirement not imposed above. When this holds we obtain

$$
\boldsymbol{\xi}=D^{-1}\left(\boldsymbol{\gamma}-C^{T} \mathbf{x}\right), \quad\left(A-B D^{-1} C^{T}\right) \mathbf{x}=\mathbf{g}-B D^{-1} \boldsymbol{\gamma} .
$$

Note that the coefficient matrix in $(2.5 b)$ is just the Schür complement of $D$ in $\mathscr{A}$. Thus if, as we have just assumed, $D$ is nonsingular this Schür complement is also nonsingular. But we also note that this coefficient matrix is an at most rank $\nu$ modification of $A$; that is $B D^{-1} C^{T}$ has the structure required to apply the Woodbury formula [4, p. 124] to solve (2.5b). Specifically if $\boldsymbol{A}$ is nonsingular then

$$
\left(A-B D^{-1} C^{T}\right)^{-1}=A^{-1}+A^{-1} B T C^{T} A^{-1},
$$

where

$$
T=\left(D-C^{T} A^{-1} B\right)^{-1} \text {. }
$$

The inverse in (2.6b) exists since $T^{-1}$ is just the Schür complement of $A$ in $\mathscr{A}$. However the application of $(2.5 \mathrm{a}, \mathrm{b})$ and $(2.6 \mathrm{a}, \mathrm{b})$ is more restrictive and more costly than the bordering algorithm and so we do not consider it further here. 
3. Singular $\boldsymbol{A}$, nonsingular $\mathscr{A}$. We now turn to the case of singular $\boldsymbol{A}$ with nullity $\nu$. Equivalently we can assume that

$(3.1 \mathrm{a}, \mathrm{b}, \mathrm{c})$

$$
\begin{aligned}
& \operatorname{dim} \mathcal{N}(\boldsymbol{A})=\nu, \\
& \mathcal{N}(\boldsymbol{A})=\operatorname{span}\left\{\boldsymbol{\phi}_{1}, \cdots, \boldsymbol{\phi}_{\nu}\right\}, \quad \Phi \equiv\left(\boldsymbol{\phi}_{1} \cdots \boldsymbol{\phi}_{\nu}\right), \\
& \mathcal{N}\left(\boldsymbol{A}^{T}\right)=\operatorname{span}\left\{\boldsymbol{\psi}_{1}, \cdots, \boldsymbol{\psi}_{\nu}\right\}, \quad \Psi \equiv\left(\boldsymbol{\psi}_{1} \cdots \boldsymbol{\psi}_{\nu}\right) .
\end{aligned}
$$

Under condition (3.1a) the matrix $\mathscr{A}$ of $(1.1)$ is nonsingular if and only if (see [1, Appendix II])
$\left.\mathrm{c}_{0}\right) \operatorname{dim} \mathscr{R}(B)=\nu$,
c $\left.c_{1}\right) \quad \mathscr{R}(B) \cap \mathscr{R}(A)=0$,
$\left.\mathrm{c}_{2}\right) \operatorname{dim} \mathscr{R}\left(C^{T}\right)=\nu$,
c 3$) \quad \mathcal{N}\left(C^{T}\right) \cap \mathcal{N}(A)=0$.

Using the $N \times \nu$ matrices $\Phi$ and $\Psi$ of basis vectors introduced in $(3.1 \mathrm{~b}, \mathrm{c})$ we can reformulate (3.2) in the equivalent form:

$$
\begin{aligned}
& \Psi^{T} B \text { is nonsingular } \Leftrightarrow\left(3.2 \mathrm{c}_{0}, \mathrm{c}_{1}\right), \\
& C^{T} \Phi \text { is nonsingular } \Leftrightarrow\left(3.2 \mathrm{c}_{2}, \mathrm{c}_{3}\right) .
\end{aligned}
$$

The proofs of the indicated equivalences are exercises in basic linear algebra.

To solve (1.1) in this case we rewrite the system as

$$
A \mathbf{x}_{0}+B \xi_{0}=\mathbf{g}, \quad C^{T} \mathbf{x}_{0}+D \xi_{0}=\gamma .
$$

Multiply (3.4a) by $\Psi^{T}$ and use (3.3a) to get

$$
\xi_{0}=\left(\Psi^{T} B\right)^{-1}\left(\Psi^{T} \mathbf{g}\right)
$$

With this value of $\xi_{0}$ in (3.4a) we obtain

$$
A \mathbf{x}_{0}=\mathbf{g}-B\left(\Psi^{T} B\right)^{-1} \Psi^{T} \mathbf{g} .
$$

This clearly has a solution since the right-hand side is in $\mathscr{R}(A)$. The general solution of $(3.6)$ is

$$
\mathbf{x}_{0}=\mathbf{x}^{p}+\Phi \zeta_{0}
$$

where $\mathbf{x}^{p}$ is any particular solution and $\zeta_{0} \in \mathbb{R}^{\nu}$ is arbitrary. Using this in (3.4b) we obtain on recalling $(3.3 b)$ the unique value for $\zeta_{0}$ :

$$
\zeta_{0}=\left(C^{T} \Phi\right)^{-1}\left[\gamma-D \xi_{0}-C^{T} \mathbf{x}^{p}\right] .
$$

The unique solution of $(3.4 \mathrm{a}, \mathrm{b})$ is thus given in (3.5) and $(3.7 \mathrm{a}, \mathrm{b})$.

To evaluate this solution representation we need $\Phi, \Psi, \mathbf{x}^{p}, \xi_{0}$ and $\zeta_{0}$. Again for $N \gg \nu$ the work in solving for the $\nu$-vectors $\xi_{0}$ and $\zeta_{0}$ is negligible compared to that in solving for the $2 \nu+1$ vectors in $\mathbb{R}^{N}$. We turn next to the determination of the null vectors.

4. Right and left null vectors. To compute the right and left null vectors of $A$ when (3.1) holds we must use some form of full pivoting. Thus with appropriate permutation matrices $P$ and $Q$, corresponding to row and column interchanges in $A$, we must work with

$$
\bar{A} \equiv P A Q
$$

To avoid notational complexity we will, as usual, assume that the indicated interchanges have already been made in the systems (1.1) and (2.1a, b) and thus use $A$ rather than 
$\bar{A}$. Then we may assume that $A$ can be factored, via Gauss elimination, in the form

$$
A=L U \equiv\left(\begin{array}{cc}
L_{r} & 0_{r \nu} \\
L_{\nu r} & I_{\nu}
\end{array}\right)\left(\begin{array}{cc}
U_{r} & U_{r \nu} \\
0_{\nu r} & \varepsilon_{\nu}
\end{array}\right) .
$$

Here, $r+\nu=N, L_{r}$ and $U_{r}$ are nonsingular $r \times r$ matrices, $I_{\nu}$ and $\varepsilon_{\nu}$ are $\nu \times \nu$ matrices ( $I_{\nu}$ the identity), $0_{r \nu}$ and $0_{\nu r}^{T}$ are $r \times \nu$ matrices of zeros, $U_{r \nu}$ is $r \times \nu$ and $L_{\nu r}$ is $\nu \times r$. For exact calculations, which we assume for this section

$$
\varepsilon_{\nu} \equiv 0_{\nu}
$$

The matrices $L_{r}$ and $U_{r}$ are triangular with forms

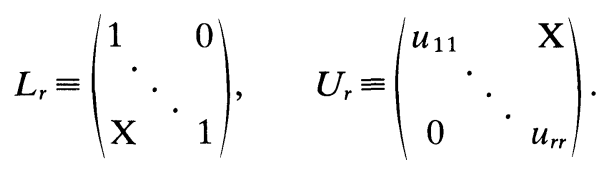

In addition $u_{11} \cdots u_{r r} \neq 0$.

To find the right null vectors we note that

$$
\boldsymbol{A} \boldsymbol{\phi}=\mathbf{0} \text { if and only if } U \boldsymbol{\phi}=\mathbf{0} .
$$

With the $\nu$ unit vectors $\mathbf{e}_{j} \in \mathbb{R}^{\nu}, 1 \leqq j \leqq \nu$, we seek vectors $\hat{\boldsymbol{\phi}}_{j} \in \mathbb{R}^{r}$ such that

$$
\boldsymbol{\phi}_{j}=a_{j}\left(\begin{array}{c}
\hat{\boldsymbol{\phi}}_{j} \\
-\mathbf{e}_{j}
\end{array}\right), \quad 1 \leqq j \leqq \nu,
$$

are right null vectors of $A$. We find that this is the case for $a_{j} \neq 0$ provided the $\hat{\boldsymbol{\phi}}_{j}$ satisfy

$$
U_{r} \hat{\boldsymbol{\phi}}_{j}=U_{r \nu} \mathbf{e}_{j}, \quad 1 \leqq j \leqq \nu .
$$

Thus a set of $\nu$ independent right null vectors of $A$ is given by

$$
\Phi \equiv\left(\begin{array}{c}
U_{r}^{-1} U_{r \nu} \\
-I_{\nu}
\end{array}\right)
$$

Similarly the left null vectors of $A$ satisfy

$$
A^{T} \boldsymbol{\psi}=\mathbf{0} \text { if and only if } L^{T} \boldsymbol{\psi}=\left(\begin{array}{l}
\mathbf{0} \\
\mathbf{e}
\end{array}\right),
$$

for arbitrary nontrivial $\mathbf{e} \in \mathbb{R}^{\nu}$. Thus if we choose for $\mathbf{e}$ the basis $\left\{\mathbf{e}_{j}\right\}$ for $\mathbb{R}^{\nu}$ we obtain the set of $\nu$ independent left null vectors

$$
\Psi \equiv\left(\begin{array}{c}
\left(L_{r}^{T}\right)^{-1} L_{\nu r}^{T} \\
-I_{\nu}
\end{array}\right) .
$$

Note that $\Phi$ and $\Psi$ are each determined by solving only triangular systems. Thus both nullspaces are obtained using only $\nu$ backsolves for the factorization $L_{r} U_{r}$.

5. Practical calculations: almost singular $\boldsymbol{A}$. In finite precision calculations, with $\boldsymbol{A}$ as in $\S 4$, we do not get $\varepsilon_{\nu} \equiv 0_{\nu}$ as assumed in (4.2). If $\boldsymbol{A}$ is appropriately scaled, then with full pivoting in $t$-digit floating arithmetic we assume an estimate of the form [10]

$$
\left\|\varepsilon_{\nu}\right\| \leqq\|A\| \times 10^{-t} \equiv \varepsilon_{0} .
$$

Indeed all the nonidentically zero elements (i.e., $0_{\nu r}$ and $0_{r \nu}$ ) and nondiagonal elements of $L_{r}$ and $I_{\nu}$ have errors that can be bounded by the same quantity. 
To assure that our procedures and analysis are applicable we must assume that the factorization procedure can, at least, correctly determine the nullity. A reasonably simple test that we have found works well in many applications (see $\S 6$ ) is to set $\nu=n$ when

$$
\max _{\substack{i>N-n \\ j>N-n}}\left|a_{i j}^{(N-n)}\right|<\delta \frac{\left|a_{N-n, N-n}^{(N-n)}\right|^{2}}{\left|a_{N-n-1, N-n-1}^{(N-n-1)}\right|^{(N-n)}} .
$$

Here we employ the usual Gaussian elimination notation $a_{i j}^{(k)}$ for the $k$ th stage of the elimination. We have used $\delta$ in $\left[10^{-3}, 10^{-2}\right]$ for many calculations. There are more robust procedures for rank determination than Gauss elimination, such as $Q R$ - and $S V$-decompositions. However for the differential equations applications these other procedures are prohibitively expensive while Gaussian elimination does not suffer from the pathological cases designed to show that it does not always work.

Further we shall require that after the numerically factored form (4.1) is obtained it satisfies

$$
\min _{j \leqq r}\left|u_{i j}\right| \gg \varepsilon_{0}
$$

say, for example, $\left|\varepsilon_{0} / u_{j j}\right| \leqq \delta$.

Finally we require for the analysis of this section that the matrix

$$
\varepsilon_{\nu} \equiv\left(a_{i j}^{(r)}\right), \quad r<i \leqq N, \quad r<j \leqq N,
$$

is nonsingular. Of course this would be the generic case if the elements $a_{i j}^{(r)}$ were truly random roundoff. But we can insure this in the actual calculations by setting

$$
\varepsilon I_{\nu} \Rightarrow \varepsilon_{\nu}
$$

where, say,

$$
\varepsilon=\frac{1}{\nu}\left\|\left(a_{i j}^{(r)}\right)\right\|_{\infty}
$$

With the above assumptions and the assurance that (5.4a) holds, we proceed to examine the bordering algorithm $(2.1 \mathrm{a}, \mathrm{b})-(2.3)$ using the factorization (4.1) and shall see how it relates to the solution in $\S 3$. We neglect for the moment all errors in the factorization save those of $\varepsilon_{\nu}$. At the end of our analysis we easily include the effects of all the errors. To conform to the partitioning in (4.1) it is useful to introduce the notation

$$
V \equiv\left(\begin{array}{c}
V_{r \nu} \\
V_{\nu}
\end{array}\right), \quad B \equiv\left(\begin{array}{c}
B_{r \nu} \\
B_{\nu}
\end{array}\right), \quad \mathbf{g} \equiv\left(\begin{array}{c}
\mathbf{g}_{r} \\
\mathbf{g}_{\nu}
\end{array}\right), \quad \mathbf{w} \equiv\left(\begin{array}{c}
\mathbf{w}_{r} \\
\mathbf{w}_{\nu}
\end{array}\right)
$$

Specifically, $V_{r \nu}$ and $B_{r \nu}$ are $r \times \nu, V_{\nu}$ and $B_{\nu}$ are $\nu \times \nu, \mathbf{g}_{r}$ and $\mathbf{w}_{r} \in \mathbb{R}^{r}, \mathbf{g}_{\nu}$ and $\mathbf{w}_{\nu} \in \mathbb{R}^{\nu}$. With (5.5), (4.1) and (4.4a, b) we obtain from (2.1a):

$$
V=\left(\begin{array}{c}
\left(L_{r} U_{r}\right)^{-1} B_{r \nu} \\
0_{\nu}
\end{array}\right)-\Phi V_{\nu} \equiv V^{p}-\Phi V_{\nu}, \quad \varepsilon_{\nu} V_{\nu}=-\Psi^{T} B
$$

and from $(2.1 b)$

$$
\mathbf{w}=\left(\begin{array}{c}
\left(L_{r} U_{r}\right)^{-1} \mathbf{g}_{r} \\
\mathbf{0}_{\nu}
\end{array}\right)-\Phi \mathbf{w}_{\nu} \equiv \mathbf{w}^{p}-\Phi \mathbf{w}_{\nu}, \quad \varepsilon_{\nu} \mathbf{w}_{\nu}=-\Psi^{T} \mathbf{g}
$$


Since $\varepsilon_{\nu}$ is assumed nonsingular $V_{\nu}$ and $\mathbf{w}_{\nu}$ are uniquely determined. We use (5.6a) and (5.7a) in (2.2) to get

$$
\left(D-C^{T} V^{P}+C^{T} \Phi V_{\nu}\right) \xi=\gamma-C^{T} \mathbf{w}^{p}+C^{T} \Phi \mathbf{w}_{\nu} .
$$

Multiply this by $\left(\Psi^{T} B\right)^{-1} \varepsilon_{\nu}\left(C^{T} \Phi\right)^{-1}$, whose existence is assured by (3.3a, b), and use $(5.6 \mathrm{~b})$ and $(5.7 \mathrm{~b})$ to write the result as

$$
\xi=\left(\Psi^{T} B\right)^{-1}\left\{\left(\Psi^{T} \mathbf{g}\right)-\varepsilon_{\nu}\left(C^{T} \Phi\right)^{-1}\left[\boldsymbol{\gamma}-C^{T} \mathbf{w}^{p}-\left(D-C^{T} V^{p}\right) \xi\right]\right\} .
$$

Recalling (3.5) and the fact that $\left\|\varepsilon_{\nu}\right\| \ll 1$, the above implies

$$
\boldsymbol{\xi}=\boldsymbol{\xi}_{0}+\mathscr{O}\left(\varepsilon_{\nu}\right) \text {. }
$$

From (5.6b) and (5.7b) we get with the aid of (5.8)

$$
\varepsilon_{\nu}\left(\mathbf{w}_{\nu}-V_{\nu} \xi\right)=-\varepsilon_{\nu}\left(C^{T} \Phi\right)^{-1}\left[\gamma-D \xi-C^{T}\left(\mathbf{w}^{p}-V^{p} \xi\right)\right] .
$$

After cancelling the $\varepsilon_{\nu}$ factor above, we use (5.6a) and (5.7a) to get from (2.3)

$$
\mathbf{x}=\mathbf{w}-V \boldsymbol{\xi}=\left(\mathbf{w}^{p}-V^{p} \boldsymbol{\xi}\right)+\Phi\left(C^{T} \Phi\right)^{-1}\left[\boldsymbol{\gamma}-D \boldsymbol{\xi}-C^{T}\left(\mathbf{w}^{p}-V^{p} \boldsymbol{\xi}\right)\right] .
$$

Setting

$$
\mathbf{x}^{p} \equiv \mathbf{w}^{p}-V^{p} \boldsymbol{\xi}_{0}
$$

and using (5.9) we find on comparison with $(3.7 \mathrm{a}, \mathrm{b})$ that

$$
\mathbf{x}=\mathbf{x}_{0}+\mathcal{O}\left(\varepsilon_{\nu}\right) \text {. }
$$

Thus we conclude from (5.9) and (5.11), that the bordering algorithm (2.1a, b)(2.3) applied to solve (1.1) with singular $A$ satisfying (3.1) yields an $\mathscr{O}\left(\varepsilon_{\nu}\right)$ accurate solution provided an $L U$-factorization is used to yield (4.1) with $\varepsilon_{\nu}$ nonsingular and satisfying (5.1) and (5.3). We must observe that since (5.3) holds the errors in computing $\Phi, \Psi, V$ and $\mathbf{w}$ are also at most $\mathcal{O}\left(\varepsilon_{0}\right)$ and so the estimates in (5.9) and (5.11) remain valid when these inexact values are used in the above derivation.

When $A$ is singular or near singular and we use the bordering algorithm, even with a good pivot strategy, some loss in accuracy is to be expected. This occurs when forming $\mathbf{x}$ as in (2.3) and is due to cancellation of leading digits. One way to circumvent these errors is to use the "singular $A$ algorithm" given by (3.5) and (3.7). This can be quite practical when $A$ is sensed to be near singular since the bases of null vectors $\Phi$ and $\Psi$ are easily determined as in $(4.4 \mathrm{a}, \mathrm{b})$.

6. Path following applications. The computational linear algebra problems discussed above were in fact motivated by the path following applications we now describe. We shall also show that when these applications come from consistent, stable, discrete approximations to a broad class of differential equation problems then very efficient partial pivoting procedures can be used for the Gaussian elimination to determine the nullity and the $L U$-factorization of (4.1). Full pivoting need only be invoked in processing the final "block" in the special banded or block tridiagonal systems that arise.

We assume that some nonlinear operator equation has been discretized and that the resulting finite dimensional problem has the form

$$
\mathbf{G}(\mathbf{u}, \lambda)=\mathbf{0} .
$$

Here $\mathbf{G}: \mathbb{R}^{N+1} \rightarrow \mathbb{R}^{N}$ is an appropriately smooth function, $\mathbf{u} \in \mathbb{R}^{N}$ and $\lambda \in \mathbb{R}$. We are concerned with computing families or "paths" of solutions, $(\mathbf{u}, \lambda)$, of $(6.1)$. One of 
the most effective ways to do this is known as Euler-Newton continuation. Thus if $(\mathbf{u}(\lambda), \lambda)$ represents an arc of solutions of (6.1) the tangent to this arc is in the direction $\left(\mathbf{u}_{\lambda}(\lambda), 1\right)$, where $\mathbf{u}_{\lambda}$ satisfies

$$
\mathbf{G}_{\mathbf{u}}(\mathbf{u}(\lambda), \lambda) \mathbf{u}_{\lambda}=-\mathbf{G}_{\lambda}(\mathbf{u}(\lambda), \lambda),
$$

then a good approximation to the solution $(\mathbf{u}(\lambda+\Delta \lambda), \lambda+\Delta \lambda)$ is given by

$$
\mathbf{u}^{0}(\lambda+\Delta \lambda)=\mathbf{u}(\lambda)+\Delta \lambda \mathbf{u}_{\lambda}(\lambda) .
$$

This is the approximation obtained by using one "Euler step" to solve (6.2) numerically. Now we use this approximation as the first iterate in Newton's method to solve (6.1) at $\lambda+\Delta \lambda$ :

$$
\mathbf{G}_{\mathbf{u}}^{\nu} \Delta \mathbf{u}^{\nu}=-\mathbf{G}_{\lambda}^{\nu}, \quad \mathbf{u}^{\nu+1}=\mathbf{u}^{\nu}+\Delta \mathbf{u}^{\nu} .
$$

Here we have used $\mathbf{G}_{\mathbf{u}}^{\nu} \equiv \mathbf{G}_{\mathbf{u}}\left(\mathbf{u}^{\nu}(\lambda+\Delta \lambda), \lambda+\Delta \lambda\right), \mathbf{G}_{\lambda}^{\nu} \equiv \mathbf{G}_{\lambda}\left(\mathbf{u}^{\nu}(\lambda+\Delta \lambda), \lambda+\Delta \lambda\right)$.

This procedure generally works extremely well. But during the course of the continuation or path following, difficulties occur if the $N \times N$ Jacobian matrix $\mathbf{G}_{\mathbf{u}}$ becomes singular. Such singular points are not uncommon and indeed they can be extremely important in the applications. Their most frequent occurrence is at limit points, say $\left(\mathbf{u}_{0}, \lambda_{0}\right)$ where

$$
(6.5 \mathrm{a}, \mathrm{b}) \quad \operatorname{dim} \mathcal{N}\left(\mathbf{G}_{\mathbf{u}}\left(\mathbf{u}_{0}, \lambda_{0}\right)\right)=1, \quad \mathbf{G}_{\lambda}\left(\mathbf{u}_{0}, \lambda_{0}\right) \notin \operatorname{Range}\left[\mathbf{G}_{\mathbf{u}}\left(\mathbf{u}_{0}, \lambda_{0}\right)\right] .
$$

Geometrically this occurs at points where the tangent to the path becomes vertical on a versus $\lambda$ graph. At bifurcation points we also have (6.5a) but not (6.5b). In a number of other important cases the null space dimension is greater than one. These include multiple limit points [1], Hopf bifurcation and period doubling bifurcations in the study of periodic solution branches [2], fold following [3], and critical boundary paths [9]. In all of these cases there are additional parameters in the problem formulation (for example: the period, $T$, of the periodic solution, etc.) or there are natural parameters that can be introduced. Indeed the idea of introducing additional parameters leads to our current study and the application of bordering.

At limit points the difficulties are easily eliminated, in principal, by simply using some arclength-like parameter to describe the path. Thus we imagine a family or arc of solutions of (6.1) given by $(\mathbf{u}(s), \lambda(s))$ for $s \in \mathscr{I} \subset \mathbb{R}$. Let $\left(\dot{\mathbf{u}}_{0}, \dot{\lambda}_{0}\right)$ be the tangent to the solution arc for $s=s_{0}$. Then we consider the scalar constraint

$$
N(\mathbf{u}, \lambda, s) \equiv \dot{\mathbf{u}}_{0} \cdot\left(\mathbf{u}-\mathbf{u}_{0}\right)+\dot{\lambda}_{0}\left(\lambda-\lambda_{0}\right)-\left(s-s_{0}\right)=0 .
$$

Now we seek to solve (6.1) and (6.6) simultaneously for $\left|s-s_{0}\right|$ not too large. We call this procedure pseudo-arclength continuation [7], since if we let $s \rightarrow s_{0}$, then (6.6) implies

$$
\left\|\dot{\mathbf{u}}_{0}\right\|_{2}^{2}+\left|\dot{\lambda}_{0}\right|^{2}=1
$$

Thus $s-s_{0}$ in (6.6) is a local approximation to arclength.

If Newton's method is used to solve (6.1) and (6.6) simultaneously for $(\mathbf{u}(s), \lambda(s))$ we get for the Newton corrections $\left(\Delta u^{\nu}, \Delta \lambda^{\nu}\right)$ a linear system of the form (1.1) with

$$
\mathscr{A}^{\nu} \equiv\left(\begin{array}{cc}
\mathbf{G}_{\mathbf{u}}^{\nu} & \mathbf{G}_{\lambda}^{\nu} \\
\dot{\mathbf{u}}_{0}^{T} & \dot{\lambda}_{0}
\end{array}\right)
$$

When $(6.5 \mathrm{a}, \mathrm{b})$ holds we can easily show that $\mathscr{A}^{\nu}$ evaluated at $\left(\mathbf{u}_{0}, \lambda_{0}\right)$ is nonsingular. Thus for $\left(\mathbf{u}^{\nu}, \lambda^{\nu}\right)$ close to $\left(\mathbf{u}_{0}, \lambda_{0}\right), \mathscr{A}^{\nu}$ is nonsingular while $\mathbf{G}^{\nu}$ is close to the singular matrix $\mathbf{G}_{\mathbf{u}}\left(\mathbf{u}_{0}, \lambda_{0}\right)$. In this situation our analysis in $\S 5$ with $\nu=1$, is applicable to the 
solution of the Newton equations. As previously mentioned this $\nu=1$ case is also discussed in [8]. However in the higher dimensional periodic bifurcation and fold following cases [2], [3], [9], where $\nu=2$, our current analysis is also applicable. The inflation procedures used to get systems of order $N+2$ are along the same lines as the above but considerably more complicated so we do not sketch them here.

To show how partial pivoting can be justified for an important class of singular $\boldsymbol{A}=\mathbf{G}_{\mathbf{u}}$ we consider the linear boundary value problem

$(6.8 \mathrm{a}, \mathrm{b}, \mathrm{c})$

$$
\begin{aligned}
& B_{a}(\lambda) \mathbf{y}(a)=\boldsymbol{\beta}_{a}, \\
& \mathbf{y}^{\prime}-A(\lambda, t) \mathbf{y}=\mathbf{f}(t), \quad a<t<b, \\
& B_{b}(\lambda) \mathbf{y}(b)=\boldsymbol{\beta}_{b} .
\end{aligned}
$$

Here $\mathbf{y}, \mathbf{f} \in \mathbb{R}^{m}, \boldsymbol{B}_{a}$ is $p \times m, \boldsymbol{B}_{b}$ is $q \times m, p+q=m, \boldsymbol{\beta}_{a} \in \mathbb{R}^{p}$ and $\boldsymbol{\beta}_{b} \in \mathbb{R}^{q}$. Of course in applications this may represent the linearization of some nonlinear problem but that is not important for our current discussion. If $Y(\lambda ; t)$ is a fundamental solution matrix for $(6.8 \mathrm{~b})$ then it is well known [6] that $(6.8 \mathrm{a}, \mathrm{b}, \mathrm{c})$ has a unique solution for each $\left(\mathbf{f}, \boldsymbol{\beta}_{a}, \boldsymbol{\beta}_{b}\right)$ if and only if

$$
F(\lambda) \equiv \operatorname{det}\left(\begin{array}{l}
B_{a}(\lambda) Y(\lambda ; a) \\
B_{b}(\lambda) Y(\lambda ; b)
\end{array}\right) \neq 0
$$

Indeed the "eigenvalues," $\lambda$, of the homogeneous case of $(6.8 \mathrm{a}, \mathrm{b}, \mathrm{c})$ are just the roots of

$$
F(\lambda)=0 .
$$

If $A(\lambda, t), B_{a}(\lambda)$ and $B_{b}(\lambda)$ are analytic in $\lambda$ then the same is true of $F(\lambda)$ and we have that the eigenvalues are isolated and accumulate only at $\infty$ (or else all values are eigenvalues). We make one further assumption:

(6.10) The eigenvalues are nonconstant functions of the coefficients in $B_{b}(\lambda)$.

Suppose we approximate the solution of $(6.8 \mathrm{a}, \mathrm{b}, \mathrm{c})$ by using the Box scheme:

$(6.11 \mathrm{a}, \mathrm{b}, \mathrm{c})$

$$
\begin{aligned}
& B_{a}(\lambda) \mathbf{y}_{0}=\boldsymbol{\beta}_{a}, \\
& \frac{\mathbf{y}_{j}-\mathbf{y}_{j-1}}{h}-\boldsymbol{A}\left(\lambda, t_{j-1 / 2}\right) \frac{\mathbf{y}_{j}+\mathbf{y}_{j-1}}{2}=\mathbf{f}\left(t_{j-1 / 2}\right), \quad 1 \leqq j \leqq J, \\
& B_{b}(\lambda) \mathbf{y}_{J}=\boldsymbol{\beta}_{b} .
\end{aligned}
$$

If we order the difference equations as indicated and denote the $N=m(J+1)$ unknowns as $\mathbf{y}^{h} \equiv\left(\mathbf{y}_{0}^{T}, \cdots, \mathbf{y}_{J}^{T}\right)^{T}$, then $(6.11 \mathrm{a}, \mathrm{b}, \mathrm{c})$ can be written as

$$
\mathbb{A}_{h}(\lambda) \mathbf{y}^{h}=\mathbf{f}^{h},
$$

where $\mathbb{A}_{h}(\lambda)$ has the block tridiagonal structure

$$
\mathbb{A}_{h}(\lambda) \equiv\left[B_{j}, A_{j}, C_{j}\right]
$$

In particular,

$$
\left.B_{j} \equiv\left(-\frac{\mathrm{X}}{0}\right)_{\}}^{\} p}, \quad C_{j} \equiv\left(\frac{0}{\mathrm{X}}\right)_{\}}^{\}}\right\} \text {. }
$$

$$
A_{0}(\lambda) \equiv\left(\frac{B_{a}(\lambda)}{\mathrm{X}} \frac{\lambda}{-}\right)_{\} q}, \quad A_{J}(\lambda) \equiv\left(-\frac{\mathrm{X}}{B_{b}(\bar{\lambda})}\right)^{\} p} .
$$


Now from [6, Thm. 2.9] we conclude that: if $\lambda$ is not an eigenvalue (i.e., root of (6.9b)) then for some $h_{0}>0$ and all $h \leqq h_{0}$ the matrices $\left\{\mathbb{A}_{h}(\lambda)\right\}$ are nonsingular with

$$
\left\|\mathbb{A}_{h}^{-1}(\lambda)\right\|<K \text {. }
$$

It is further shown in $[5, \S 5]$, that when $(6.14)$ holds a restricted form of pivoting yields $L U$-factorizations of the form:

$$
\mathbb{A}_{h}(\lambda)=\left[\beta_{j}, \delta_{j}, 0\right]\left[0, \alpha_{j}, \gamma_{j}\right], \quad 0 \leqq j \leqq J,
$$

where $\alpha_{j}, \beta_{j}, \delta_{j}$ and $\gamma_{j}$ are $m \times m$ matrices. The restriction on the pivoting is such that it allows interchanges within the set of $m$ equations (6.11b) at any fixed net point (a restricted form of row-pivoting for $\mathbb{A}_{h}$ ) and it allows interchanges within the $m$ variables $\mathbf{y}_{j}$ associated at each net point (a restricted form of column-pivoting). Both of these pivoting strategies insure that the zero structure of the $B_{j}$ and $C_{j}$ are preserved in the $\beta_{j}$ and $\gamma_{j}$, respectively. To obtain factorizations of the form (4.1), (4.3) we use these techniques with $\delta_{j}$ and $\alpha_{j}$ in the triangular forms

$$
\delta_{j} \equiv\left(\begin{array}{ccc}
1 & & 0 \\
& \ddots & \\
\mathrm{X} & & 1
\end{array}\right), \quad \alpha_{j} \equiv\left(\begin{array}{lll}
x & & \mathrm{X} \\
& \ddots & \\
0 & & x
\end{array}\right) .
$$

We note, by $(6.13 \mathrm{~b})$ that the coefficients defining $B_{b}(\lambda)$ do not enter into the elimination procedure until the final $m \times m$ block is to be factored as

$$
\delta_{J} \alpha_{J}=A_{J}(\lambda)-\beta_{J} \gamma_{J-1} \equiv\left(-\frac{\mathrm{X}}{B_{b}(\lambda)}\right) .
$$

Furthermore since $\beta_{J}$ contains zeros in the last $q$ rows (as in all the $B_{j}$ of (6.13a)), the last $q$ rows on the right-hand side of (6.17) are just those of $B_{b}(\lambda)$.

Now if $\lambda$ is an eigenvalue we could invoke (6.10) to ensure that the elimination does not fail until all but the last block has been processed. This is simply done by changing the data in $B_{b}(\lambda)$ so that the current value of $\lambda$ is no longer an eigenvalue. Then the complete factorization is valid. But this uses data from $B_{b}(\lambda)$ only in the final block. Thus in the singular case (i.e., at an eigenvalue) we need not actually change any data-we merely use full pivoting in the final factorization of (6.17). A similar argument can be used to justify these techniques on discrete approximations for many other classes of functional equations including elliptic boundary value problems. The crucial requirement is some analogue of $(6.10)$ which insures that changing the boundary conditions changes the eigenvalues.

Acknowledgments. I wish to thank the referees for helpful comments on the original version of this paper.

\section{REFERENCES}

[1] D. W. Decker And H. B. Keller, Multiple limit point bifurcation, J. Math. Anal. Appl., 75 (1980), pp. $417-430$.

[2] E. Doedel, A. Jepson ANd H. B. Keller, Paths of periodic solutions and their computation, in preparation.

[3] J. FiER AND H. B. KELlER, Follow the folds, in preparation.

[4] A. S. Householder, The Theory of Matrices in Numerical Analysis, Dover, New York, 1975.

[5] H. B. KEller, Accurate difference methods for nonlinear two point boundary value problems, SIAM J. Numer. Anal., 11 (1974), pp. 305-320. 
[6] - Numerical Solution of Two Point Boundary Value Problems, CBMS Regional Conference Series in Applied Mathematics 24, Society for Industrial and Applied Mathematics, Philadelphia, 1976.

[7] - Numerical Solution of bifurcation and nonlinear eigenvalue problems, in Applications of Bifurcation Theory, P. Rabinowitz, ed., Academic Press, New York, 1977, pp. 359-384.

[8] - Practical procedures in path following near limit points, to appear in Computing Methods in Applied Sciences and Engineering, R. Glowinski and J. L. Lions, eds., North-Holland Pub. Co., Amsterdam, 1982.

[9] W. RHEINBOLDT, Computation of critical boundaries on equilibrium manifolds, SIAM J. Numer. Anal., 19 (1982), pp. 653-669.

[10] J. H. Wilkinson, Rounding Errors in Algebraic Processes, Prentice-Hall, Englewood Cliffs, NJ, 1963. 